\title{
Médicos de família encaminham menos? Impacto da formação em MFC no percentual de encaminhamentos da Atenção Primária
}

\author{
Do family doctors refer less? Impact of FCM training \\ on the rate of PHC referrals
}

Ricardo Collar Rebolho (https://orcid.org/0000-0002-7797-8037) ${ }^{1}$

Paulo Poli Neto (https://orcid.org/0000-0003-1073-6583) ${ }^{2}$

Lucas Alexandre Pedebôs (https://orcid.org/0000-0001-6663-352X) ${ }^{1}$

Leandro Pereira Garcia (https://orcid.org/0000-0002-8601-7166) ${ }^{1}$

Ana Cristina Vidor (https://orcid.org/0000-0003-2518-6414) ${ }^{1}$

${ }^{1}$ Secretaria Municipal de Saúde de Florianópolis. Av. Prof. Henrique da Silva Fontes 6100, Trindade. 88036-700 Florianópolis SC Brasil. ricardo_rebolho@ hotmail.com ${ }^{2}$ Departamento de Saúde Comunitária, Universidade Federal do Paraná. Curitiba PR Brasil.

\begin{abstract}
Objective: To evaluate the impact of family medicine residence on the PHC referral rate. Methods: This is a cross-sectional descriptive study on 375.645 visits and 34.776 referrals by 123 PHC physicians in 2016, linking the referral rate to the characteristics of doctors (gender, age, family medicine training), patients (gender and age) and service (general population and working population). Results: Family and community medicine residency training had a significant reduction in PHC referral rate (2.86\%), CI:(1.55;4.17), $p<0,0001$. This reduction persisted in the multivariate analysis, after adjusting for all the possible confounding variables. No difference was found between the referral rates of doctors with and without family and community medicine (FCM) degree. Concerning referral to specialties, doctors with FCM residence training had lower rates of referral to gynecology, psychiatry and pediatrics and higher rates of referral to ophthalmology. Conclusion: The study showed that FCM residency significantly reduced $\mathrm{PHC}$ referral rates.
\end{abstract}

Key words Family and community medicine, Primary health care, Referral and visit, Medical residency
Resumo O objetivo deste artigo é medir o impacto da formação em medicina de família e comunidade no percentual de encaminhamentos a partir da atenção primária. Estudo transversal descritivo de 375.645 consultas e 34.776 encaminhamentos realizadas por 123 médicos da atenção primária no ano de 2016 relacionando o percentual de encaminhamentos com características dos médicos (sexo, idade, formação em MFC), dos pacientes (sexo e idade) e do serviço (população pelo IBGE e população ativa). A formação em MFC por meio de residência médica apresentou uma significativa redução no percentual de encaminhamentos a partir da atenção primária (2,86\%), IC:(1,55;4,17), $p<0,0001$. Essa redução manteve-se na análise multivariada mesmo ajustando-se para todas as possiveis variáveis confundidoras. Não houve diferença na comparação do percentual de encaminhamentos entre médicos sem formação em MFC e médicos com titulação em MFC. Na análise das especialidades para as quais foram realizados os encaminhamentos, os médicos com residência em MFC encaminharam menos para ginecologia, psiquiatria e pediatria e mais para oftalmologia. O estudo mostrou que a formação em MFC por meio de residência médica acarretou significativa redução no percentual de encaminhamentos a partir da atenção primária.

Palavras-chave Medicina de família e comunidade, Atenção primária à saúde, Encaminhamento e consulta, Residência médica 


\section{Introdução}

Sabe-se que a organização dos sistemas de saúde com melhores resultados para sua população e melhor custo efetividade são aquelas pautadas por uma atenção primária forte e resolutiva ${ }^{1}$. A atenção primária funciona como porta de entrada para o sistema de saúde e como coordenadora do cuidado, tendo a atribuição de lidar com 85 a 95\% dos problemas de saúde da população. Uma das funções mais importantes da atenção primária é exercer o papel de filtro, ou gate keeper, isto é, definir quais pacientes devem ser encaminhados para avaliações pontuais ou continuadas na atenção especializada e quais podem ter toda a sua demanda naquele momento atendida ${ }^{2}$. Tal função exerce papel fundamental na segurança e na qualidade do tratamento ofertado ao usuário, uma vez que o acompanhamento unicamente por especialistas focais pode gerar prescrições excessivas, desnecessárias e potencialmente danosas, sobretudo quando esse acompanhamento acontece por médicos de diferentes especialidades concomitantemente. Ainda, a atenção especializada costuma caracterizar-se por seus maiores custos, fazendo com que um número além do ideal de encaminhamentos contribua para a redução da eficiência do sistema. No oposto, privar o usuário de obter tal avaliação pode gerar um tratamento subótimo, devendo a atenção primária estar atenta a esse equilíbrio ${ }^{3}$.

Partindo desse princípio, o Sistema Único de Saúde (SUS) brasileiro está organizado a partir dos níveis de atenção primário e serviços especializados. No nível primário, a Estratégia de Saúde da Família (ESF) é o programa responsável pela atenção básica. Neste programa, as equipes de saúde da família, formadas por médicos, enfermeiros, técnicos de enfermagem e agentes comunitários de saúde, são distribuídas por todo o território nacional nas Unidades Básicas de Saúde (UBS), que são a porta de entrada para o sistema de saúde. É responsabilidade dessas equipes o cuidado da saúde da população adscrita, que no sistema brasileiro é dividida com base territorial e cada equipe é responsável pelo atendimento de 2 a 3,5 mil pessoas. ${ }^{4}$. Cabe aos profissionais médicos dessas equipes definir quais pacientes necessitam ou não de encaminhamento para os serviços especializados, e só a partir deste encaminhamento o paciente acessará os demais níveis do sistema de saúde ${ }^{2}$.

Para que este profissional seja capaz de exercer adequadamente sua função de gate keeper e responder adequadamente a 85 a $95 \%$ das de- mandas de saúde dos usuários, é importante que ele tenha formação e conhecimento adequados para tal, caso contrário a organização do sistema de saúde se torna mais dispendiosa e descoordenada $^{5}$. No Brasil, não é necessária nenhuma formação além da graduação em medicina para atuar na atenção primária, o que faz com que muitos profissionais sem essa formação específica acabem ocupando essa função essencial no sistema. Sabe-se que os sistemas de saúde com melhores resultados para suas populações são aqueles em que a atenção primária é efetivamente resolutiva e desempenha bem sua função de porta de entrada, encaminhando os casos necessários ${ }^{2}$. Para isso, os profissionais que ali atuam possuem formação para tal, passando por programas de residência após a graduação. No Brasil, a medicina de família e comunidade é a especialidade que reúne todos os conhecimentos desejáveis para atuar na atenção primária à saúde, e a residência em medicina de família e comunidade é o programa através do qual o egresso da graduação adquire esses conhecimentos ${ }^{6}$. Porém, como a oferta desses profissionais no mercado de trabalho ainda está bem abaixo do necessário, por diversos fatores, são minoria os profissionais que atuam na atenção primária com essa formação ${ }^{7}$.

Florianópolis é considerada a capital com a melhor rede de atenção primária à saúde do Brasil, pois, entre outros fatores, possui cobertura populacional de $100 \%$ pelas ESFs e possui $70 \%$ de médicos com formação em medicina de família e comunidade atuando na rede ${ }^{8}$. Apesar disso, $30 \%$ dos médicos que atuam na rede não possuem essa formação.

Vários estudos ao redor do mundo avaliaram o percentual de encaminhamentos a partir da atenção primária e os fatores que influenciavam sua ocorrência ${ }^{9-15}$. Os números variavam de 5 a $30 \%$ de consultas que resultavam em encaminhamentos $^{9-15}$. Diversos fatores foram pesquisados para tentar explicar essa grande variabilidade, parte deles pode ser explicada pelas variações dos pacientes, como idade, raça, gênero, número de comorbidades ${ }^{16}$. Outra parte é devido aos fatores do médico, como idade, formação em medicina de família, forma de pagamento, tempo na mesma equipe e gênero ${ }^{17-19}$. Alguns estudos também pesquisaram a influência dos recursos locais, como número de serviços de atenção especializada disponíveis e distância até os serviços ${ }^{17-19}$.

Apesar de existirem diversos estudos em andamento avaliando o percentual de encaminhamentos para a atenção especializada no Brasil, em nossa busca não encontramos nenhum estudo 
publicado até o momento. Apesar de não haver nenhuma evidência científica do número ideal de encaminhamentos a partir da atenção primária, um estudo publicado em 1993 com mais de 1.500 médicos de 15 países da Europa ocidental, mostrou percentual de encaminhamentos variando entre 2,6 e $8,2 \%{ }^{20}$. Esse percentual pode representar um parâmetro de qualidade da atenção primária, assim como gerar um grande impacto financeiro para o sistema de saúde.

Dessa forma, o presente estudo tem como objetivo identificar a influência de fatores relacionados aos médicos, ao serviço e aos usuários no percentual de encaminhamentos à atenção especializada a partir dos atendimentos realizados na atenção primária em saúde no município de Florianópolis-SC.

\section{Métodos}

\section{População de estudo}

Foram analisados os percentuais de encaminhamentos de 123 médicos da atenção primária, que atuaram na Secretaria Municipal de Saúde de Florianópolis (SMS) no ano de 2016. Foram incluídos no estudo todos os médicos que realizaram no mínimo 500 consultas no ano de 2016 e que eram responsáveis por alguma equipe da Estratégia de Saúde da Família, este número de consultas foi escolhido como ponto de corte por ser a média das consultas realizadas por um médico no período de um mês. Não foram incluídos médicos do Núcleo de Apoio a Saúde da Família (NASF) ou médicos residentes. A equipe do médico foi considerada aquela em que estavam cadastrados a maioria dos pacientes que ele atendeu no ano de 2016.

\section{Extração dos dados}

A coleta de dados deu-se por duas estratégias: extração de dados do sistema de registro eletrônico em saúde da SMS e busca de dados complementares na internet. Do sistema de registro eletrônico em saúde, foram extraídos dados de todas as consultas realizadas em todas as UBSs da cidade de Florianópolis no ano de 2016, de acordo com a unidade de atendimento, nome do profissional, matrícula na prefeitura, número do registro do profissional, código da consulta, código do encaminhamento (quando realizado) e para quais especialidades, código, idade e sexo do usuário, área/equipe adscrita em que este está cadastrado. Além disso, foram coletados no setor de recursos humanos da Secretaria Municipal de Saúde gênero e idade dos médicos. Não se teve acesso ao prontuário dos pacientes, sendo todo o conjunto citado de dados fornecidos por terceiros da própria instituição. A estimativa populacional para cada equipe foi obtida a partir de projeção realizada pela equipe da própria Secretaria Municipal de Saúde a partir do censo demográfico do IBGE de $2010^{21}$. A busca por dados da formação do médico foi realizada de três formas: 1) Lista de titulados em todas as edições já realizadas da prova de Título de Especialista em Medicina de Família e Comunidade (TEMFC) no site da Sociedade Brasileira de Medicina de Família e Comunidade (SBMFC) $\left.)^{22} ; 2\right)$ Registro da especialidade medicina de família e comunidade no Conselho Regional de Medicina (CRM), através da busca por médicos no site do $\mathrm{CRM}^{23}$; 3) Sistema da Comissão Nacional de Residência Médica (SisCNRM) de consulta de Certificados de Residência Médica em medicina de família e comuni$\mathrm{dade}^{24}$. Foram considerados com residência todos os médicos que concluíram a residência médica em MFC até março de 2016, independente de possuir ou não título de especialista em MFC. Foram considerados com titulação os médicos sem registro de conclusão de residência médica em MFC e que foram aprovados no TEMFC até março de 2016. Foram considerados não MFC os médicos que não possuíam nem residência nem titulação em MFC até março de 2016.

\section{Análise dos dados}

O percentual de encaminhamentos foi calculado dividindo o número total de encaminhamentos pelo número total de consultas. Só foram contabilizados os encaminhamentos para especialidades médicas. A média da idade da população foi calculada somando-se a idade das pessoas atendidas pelo médico, dividida pelo número total de pacientes atendidos pelo mesmo médico, no ano de 2016. A proporção de mulheres atendidas por cada médico foi calculada de forma semelhante. A população ativa de cada equipe foi definida como o total de pessoas diferentes atendidas em consultas médicas e de enfermagem de 01/01/2015 a 31/12/2016 a partir de levantamento de dados do setor de informação em saúde.

Foram descritas as características dos médicos e pacientes de acordo com a formação médica. Para a comparação de proporções das variáveis categóricas foi utilizado o teste de qui-quadrado de Pearson. Para a comparação das médias entre 
dois ou mais grupos foi utilizado o teste ANOVA. Para correlação entre o percentual de encaminhamentos e as variáveis categóricas foi utilizado o teste $t$ de Student e para a correlação do percentual de encaminhamentos com as variáveis quantitativas foi utilizado o coeficiente de correlação de Pearson. Foram considerados os valores de $r$ $<0,25$ como sem relação e entre 0,25 e 0,5 como relação fraca. Foram considerados significantes os valores de $\mathrm{p}<0,05$. Para todas as comparações o grupo padrão foi o de médicos não MFC. Em todas as análises a variável dependente foi o percentual de encaminhamentos, enquanto as variáveis independentes foram: Formação em MFC, gênero do médico, idade do médico, proporção de mulheres na população atendida, média de idade da população atendida, população IBGE e população ativa.

Para o ajuste de possíveis variáveis confundidoras no percentual de encaminhamentos foi realizada uma regressão multivariada. Para esta análise, todas as variáveis com um $\mathrm{p} \leq 0,25$ na análise bivariada entraram no modelo multivariado, conforme recomendação de Hosmer e Lemeshow $^{25}$. Nesta análise foram comparados apenas os médicos com residência versus os sem residência, sendo também incluídos neste último grupo os médicos apenas com titulação, visto que na análise bivariada não houve diferença entre os médicos sem residência e os que possuíam apenas titulação. Após analisar o modelo inicial, diversas variáveis deixaram de ser estatisticamente significantes. Foi elaborado um novo modelo, apenas com as variáveis que mantiveram um $\mathrm{p} \leq 0,05$, e este foi o escolhido. Estas análises foram executadas utilizando-se os softwares estatísticos $\mathrm{R}$ v.3.3.2. $2^{26}$ e R-Studio v.1.0.4427.

\section{Considerações éticas}

A coleta de dados foi autorizada pela Secretaria Municipal de Saúde através da Comissão de Acompanhamento dos Projetos de Pesquisa em Saúde e aprovado pelo Comitê de Ética e Pesquisa da Secretaria de Estado da Saúde de Santa Catarina.

\section{Resultados}

A Tabela 1 mostra as características dos médicos, pacientes e serviço de acordo com a formação médica. Observamos que 47,97\% dos médicos incluídos no estudo possuíam residência em MFC, $13,82 \%$ possuíam titulação em MFC e 38,21\% não possuíam nem residência nem titulação em
MFC. Os valores de $p$ referem-se à comparação com a população padrão (não MFC).

$O$ percentual de encaminhamentos total foi de $9,57 \%$. Na Tabela 2 temos a análise bivariada do impacto de cada uma das variáveis no percentual de encaminhamentos. Na comparação entre os médicos com residência em MFC versus os com titulação em MFC, o percentual de encaminhamentos foi significativamente menor entre os primeiros $(8,06 \%$ versus $11,04 \%$, IC $95 \%$ $(1,09 ; 4,85), \mathrm{p}=0,002) . \mathrm{Na}$ análise das variáveis contínuas com o percentual de encaminhamentos houve uma correlação fraca, porém significativa, entre o percentual de encaminhamentos e a idade dos médicos, a média de idade dos pacientes e a porcentagem de mulheres na população, ou seja, quanto maior a idade dos médicos, dos pacientes ou a porcentagem de mulheres na população, maior o percentual de encaminhamentos. Das variáveis relacionadas ao serviço, tanto a população por equipe pelo IBGE quanto a população ativa não apresentaram correlação com o percentual de encaminhamentos.

Na Tabela 3 temos os coeficientes de regressão e intervalos de confiança brutos e ajustados das regressões lineares. Nesta análise, mantiveram efeito significativo no percentual de encaminhamentos apenas a média de idade dos médicos, a média de idade da população e a residência em MFC, sendo esta última responsável pelo maior impacto isolado no percentual de encaminhamentos.

$\mathrm{Na}$ Tabela 4, temos a proporção dos encaminhamentos para cada especialidade de acordo com a formação médica. As especialidades com maior proporção de encaminhamentos em cada um dos grupos são semelhantes, porém há diferença significativa entre os grupos nas seguintes especialidades: oftalmologia, ginecologia, psiquiatria e pediatria. Na comparação grupo a grupo, essa diferença se manteve significativa entre os grupos com residência em MFC versus não MFC, nessas mesmas especialidades, com um maior percentual de encaminhamentos para oftalmologia no grupo com residência em MFC e um menor percentual de encaminhamentos para ginecologia, psiquiatria e pediatria também neste grupo. Entre os grupos com titulação em MFC versus não MFC houve diferença significativa apenas na especialidade pediatria/otorrino, com uma menor proporção de encaminhamentos no grupo com titulação em MFC. Já na comparação entre os grupos com residência versus com titulação, houve diferença significativa apenas nas especialidades ginecologia e psiquiatria, ambas com uma menor proporção no grupo com residência em MFC. 
Tabela 1. Características dos médicos, pacientes e seriço de acordo com a formação médica.

\begin{tabular}{|c|c|c|c|c|c|c|}
\hline & $\begin{array}{c}\text { Total } \\
(n=123)\end{array}$ & $\begin{array}{c}\text { Não MFC } \\
(n=47)\end{array}$ & $\begin{array}{c}\text { Residência } \\
(n=59)\end{array}$ & $\mathbf{p}$ & $\begin{array}{c}\text { Titulação } \\
(\mathrm{n}=17)\end{array}$ & $\mathbf{p}$ \\
\hline \multicolumn{7}{|l|}{ Médicos } \\
\hline Mulheres & $71(57,72 \%)$ & $33(70,21 \%)$ & $29(49,15 \%)$ & 0,029 & $9(52,94 \%)$ & 0,199 \\
\hline Idade & $40,43(10,41)$ & $40,07(12,95)$ & $37,14(6,82)$ & 0,003 & $44,76(9,95)$ & 0,626 \\
\hline \multicolumn{7}{|l|}{ Pacientes } \\
\hline Porcentagem de mulheres & $61,32(2,62)$ & $61,79(2,36)$ & $60,71(2,59)$ & 0,03 & $62,14(3,06)$ & 0,627 \\
\hline Idade & $40,27(4,10)$ & $40,64(3,88)$ & $39,2(3,80)$ & 0,057 & $42,97(4,53)$ & 0,047 \\
\hline \multicolumn{7}{|l|}{ Serviço } \\
\hline População IBGE & $3756(1768)$ & $3593(1171)$ & $3395(1141)$ & 0,383 & $5458(3399)$ & 0,002 \\
\hline População ativa & $2374(823)$ & $2404(925)$ & $2326(760)$ & 0,636 & $2459(767)$ & 0827 \\
\hline
\end{tabular}

Dados em n(\%) ou média (desvio-padrão).

Tabela 2. Impacto das variáveis associadas aos médicos, pacientes e serviço no percentual de encaminhamentos.

\begin{tabular}{|c|c|c|c|c|}
\hline & $\begin{array}{c}\text { Percentual de } \\
\text { Encaminhamentos (\%) }\end{array}$ & $\mathbf{r}$ & IC $95 \%$ & $\mathbf{p}$ \\
\hline \multicolumn{5}{|l|}{ Médicos } \\
\hline Não MFC (população padrão) & 10,92 & & & \\
\hline Residência & 8,06 & & $(1,55 ; 4,17)$ & $<0,0001$ \\
\hline Titulação & 11,04 & & $(-2,25 ; 2,02)$ & 0,916 \\
\hline Homem X Mulher & $8,76 \times 10,16$ & & $(0,05 ; 2,74)$ & 0,042 \\
\hline Idade & & 0,26 & & 0,003 \\
\hline \multicolumn{5}{|l|}{ Pacientes } \\
\hline Porcentagem de mulheres na população & & 0,28 & & 0,002 \\
\hline Idade & & 0,36 & & $<0,0001$ \\
\hline \multicolumn{5}{|l|}{ Serviço } \\
\hline População IBGE & & 0,08 & & 0,361 \\
\hline População ativa & & 0,14 & & 0,119 \\
\hline
\end{tabular}

Tabela 3. Coeficientes de regressão e intervalos de confiança brutos e ajustados das regressões lineares.

\begin{tabular}{|c|c|c|c|c|}
\hline Variável & $\beta_{1 \text { brute }}(\mathrm{IC} 95 \%)$ & $p$ & $\beta_{1 \text { ajustado }}($ IC95\%) & $p$ \\
\hline \multicolumn{5}{|l|}{ Gênero dos médicos } \\
\hline Feminino & 1 & & & \\
\hline Masculino & $-1,26(-2,60 ; 0,05)$ & & 0,06 & \\
\hline Média de idade dos médicos & $0,10(0,04 ; 0,16)$ & $<0,01$ & $0.06(0,002 ; 0,12)$ & 0,04 \\
\hline \multicolumn{5}{|l|}{ Residência Médica } \\
\hline Não & 1 & & 1 & \\
\hline Sim & $-2.75(-4,00 ;-1,50)$ & $<0,01$ & $-1,89(-3,16 ;-0,62)$ & $<0,01$ \\
\hline \multicolumn{5}{|l|}{ População } \\
\hline Percentual mulheres & $0,36(0,11 ; 0,60)$ & $<0,01$ & & \\
\hline Média de idade & $0,30(0,14 ; 0,45)$ & $<0,01$ & $0,22(0,07 ; 0,37)$ & $<0,01$ \\
\hline \multicolumn{5}{|l|}{ Serviço } \\
\hline População IBGE & $\begin{array}{r}0,0003 \\
(-0,0002 ; 0,0007)\end{array}$ & 0,25 & & \\
\hline População ativa & $\begin{array}{r}0,0008 \\
(-0,00004 ; 0,002)\end{array}$ & 0,06 & & \\
\hline
\end{tabular}

Legenda: $\beta_{1}=$ coeficientes de regressão, IC $(\mathbf{9 5} \%)$ = Intervalos de confiança de 95\%, $\boldsymbol{p}=$ probabilidade 
Tabela 4. Proporção dos encaminhamentos para cada especialidade de acordo com a formação médica.

\begin{tabular}{lrrrrr}
\hline & Total & Não MFC & Residência & Titulação & p \\
\hline Oftalmologia & $29,41 \%$ & $26,19 \%$ & $32,42 \%$ & $27,89 \%$ & $<0,0001$ \\
Ortopedia & $6,99 \%$ & $7,34 \%$ & $6,67 \%$ & $7,09 \%$ & 0,63 \\
Ginecologia & $6,54 \%$ & $7,02 \%$ & $5,42 \%$ & $9,07 \%$ & 0,003 \\
Cardiologia & $5,85 \%$ & $6,32 \%$ & $5,54 \%$ & $5,60 \%$ & 0,432 \\
Otorrinolaringologia & $4,66 \%$ & $4,66 \%$ & $4,88 \%$ & $3,91 \%$ & 0,269 \\
Peq. Cirurgias & $4,64 \%$ & $5,16 \%$ & $4,24 \%$ & $4,61 \%$ & 0,203 \\
Dermatologia & $4,58 \%$ & $4,32 \%$ & $4,81 \%$ & $4,47 \%$ & 0,68 \\
Urologia & $4,13 \%$ & $4,37 \%$ & $4,04 \%$ & $3,77 \%$ & 0,473 \\
Cirurgia Vascular & $3,62 \%$ & $3,64 \%$ & $3,68 \%$ & $3,33 \%$ & 0,755 \\
Neurologia & $3,27 \%$ & $3,61 \%$ & $2,99 \%$ & $3,27 \%$ & 0,204 \\
Cirurgia Geral & $3,27 \%$ & $3,09 \%$ & $3,51 \%$ & $2,95 \%$ & 0,368 \\
Acupuntura & $3,06 \%$ & $2,80 \%$ & $3,26 \%$ & $3,09 \%$ & 0,744 \\
Endocrinologia & $2,44 \%$ & $2,39 \%$ & $2,33 \%$ & $3,00 \%$ & 0,253 \\
Infectologia & $2,05 \%$ & $2,12 \%$ & $2,00 \%$ & $2,05 \%$ & 0,926 \\
Proctologia & $1,98 \%$ & $1,90 \%$ & $2,09 \%$ & $1,88 \%$ & 0,73 \\
Psiquiatria & $1,86 \%$ & $2,57 \%$ & $1,16 \%$ & $2,35 \%$ & 0,018 \\
Gastroenterologia & $1,61 \%$ & $1,84 \%$ & $1,50 \%$ & $1,38 \%$ & 0,313 \\
Obstetrícia/Gravidez Alto Risco & $1,49 \%$ & $1,44 \%$ & $1,50 \%$ & $1,65 \%$ & 0,85 \\
Reumatologia & $1,46 \%$ & $1,49 \%$ & $1,37 \%$ & $1,64 \%$ & 0,65 \\
Pneumologia & $1,44 \%$ & $1,45 \%$ & $1,39 \%$ & $1,56 \%$ & 0,891 \\
Oftalmo/Pediatria & $1,42 \%$ & $1,28 \%$ & $1,59 \%$ & $1,25 \%$ & 0,397 \\
Mastologia & $1,19 \%$ & $1,09 \%$ & $1,17 \%$ & $1,55 \%$ & 0,343 \\
Pediatria/Otorrino & $1,16 \%$ & $1,44 \%$ & $1,05 \%$ & $0,78 \%$ & 0,085 \\
Nefrologia & $0,96 \%$ & $0,95 \%$ & $0,97 \%$ & $0,99 \%$ & 0,986 \\
Pediatria & $0,91 \%$ & $1,56 \%$ & $0,41 \%$ & $0,88 \%$ & 0,005 \\
\hline
\end{tabular}

\section{Discussão}

O estudo demonstrou que a formação em MFC através da residência médica reduziu significativamente o percentual de encaminhamentos da atenção primária para a atenção especializada. Este impacto manteve-se, mesmo com os ajustes para as possíveis variáveis confundidoras analisadas. Podemos levantar algumas hipóteses para a explicação dessa diferença, entre elas o fato da residência em MFC contemplar um período de atuação de dois anos, sob supervisão de outro MFC mais experiente, nos quais o residente adquire capacidade para lidar com a maior parte dos problemas de saúde apresentados pela população, aumentando seu poder de resolução. Além disso, o residente aprende os princípios da MFC e pode aplicá-los na sua prática, como a longitudinalidade, em que desenvolve um vínculo com o paciente e poderá acompanha-lo ao longo de um período de tempo maior que poderá ser útil para definir quais casos realmente precisam de um encaminhamento e quais podem ser resolvi- dos na atenção primária. Também o princípio do acesso, amplamente discutido nos programas de residência em MFC, em que o residente valoriza a importância de os pacientes terem acesso fácil e em tempo oportuno ao seu médico, e que em situações de agravamento de seus problemas de saúde, poderão ser atendidos prontamente quando necessário.

As especialidades com maior proporção de encaminhamentos são semelhantes, mas há diferença significativa em algumas especialidades. O grupo com residência em MFC apresentou uma proporção significativamente maior de encaminhamentos para oftalmologia, o que pode significar um parâmetro de melhor qualidade do cuidado de pacientes crônicos neste grupo, devido à necessidade de encaminhamento anual para oftalmologia nos pacientes hipertensos e diabéticos. Já a menor proporção de encaminhamentos para ginecologia, psiquiatria e pediatria neste mesmo grupo também pode estar relacionada a uma maior resolutividade das queixas relacionadas a estas especialidades, talvez devido a compe- 
tências desenvolvidas durante o treinamento da residência. Vale ressaltar que essas três especialidades podem compor o Núcleo de Apoio a Saúde da Família (NASF) e seria interessante avaliar a presença delas nas equipes do NASF dos centros de saúde onde esses médicos trabalhavam e também se a formação em MFC tem impacto no trabalho em equipe realizado em conjunto com esses profissionais.

$\mathrm{O}$ estudo também mostrou que a titulação em MFC não apresentou impacto no percentual de encaminhamentos. Importante notar também que não houve diferença significativa na proporção de encaminhamentos para cada especialidade na comparação entre o grupo com titulação em MFC versus o grupo não MFC, exceto na especialidade pediatria/otorrino, o que pode significar que a titulação em MFC não tenha impacto na qualidade do cuidado prestado por estes médicos.

As principais fortalezas do estudo são: 1. A avaliação de um grande número de consultas (375.645) e de encaminhamentos (34.776); 2. A confiabilidade dos dados, pois todas as consultas e encaminhamentos são necessariamente realizados através do sistema informatizado; 3 . A presença de bom número de médicos nos diferentes grupos de acordo com a formação; 4 . A possibilidade de aferição de algumas variáveis confundidoras dos médicos, pacientes e do serviço.

Algumas limitações do estudo são: 1. A não avaliação de outros possíveis confundidores como: tempo de formado do médico, tempo na mesma equipe, renda da população e número de comorbidades da população; 2 . A não avaliação de se os encaminhamentos foram adequados ou não. Um estudo holandês ${ }^{28}$, por exemplo, comparou a proporção de encaminhamentos adequados de dois médicos com altos percentuais de encaminhamentos e de dois com baixos percentuais de encaminhamento e não encontrou diferença entre elas. $\mathrm{O}$ que mostra que um alto percentual de encaminhamentos não necessariamente está relacionado com encaminhamentos inadequados ou evitáveis.

O percentual de encaminhamentos encontrado neste estudo foi semelhante ao descrito na literatura, que varia de 5 a 30\% $\%^{9-15,29,30}$. Assim como em outros estudos ${ }^{11,13,17,18}$, a maior média de idade e maior proporção de mulheres na população estão relacionadas a um maior percentual de encaminhamentos. Também já foi demonstrado, em outros estudos, que médicas do sexo feminino apresentam maior percentual de encaminhamentos ${ }^{9,13,14,17}$. O aumento do percentual de encaminhamentos com o aumento da idade dos médicos encontrado neste estudo já foi descrito na literatura, porém os achados são conflitantes e não há uma explicação plausível ${ }^{9,16,17}$.

Um estudo norueguês ${ }^{14}$ encontrou resultados semelhantes quanto à formação em MFC, com menor percentual de encaminhamentos entre os médicos com residência em MFC comparados aos sem essa formação (12,5\% versus $14,9 \%$ ). Outro estudo tailandês ${ }^{31}$ também encontrou um menor percentual de encaminhamentos dos médicos com residência em MFC comparado aos médicos sem esse treinamento ( $14 \%$ versus 24\%).

Diferente deste estudo, um artigo canaden$\mathrm{se}^{19}$ encontrou que as especialidades com maior número de encaminhamentos a partir da atenção primária foram: Gastroenterologia (11\%), Ginecologia (10\%), Dermatologia (9\%), Cirurgia geral $(9 \%)$, Ortopedia (7\%), Otorrinolaringologia (6\%), Oftalmologia (4\%), Alergologia e imunologia (4\%), Cardiologia (4\%) e Cirurgia Plástica (4\%). Esses números podem ser influenciados pelo fato de no Canadá os pacientes acessarem diretamente o optometrista para refração, o que diminui consideravelmente o número de encaminhamentos para a oftalmologia.

O presente estudo reafirma a importância da formação em medicina de família e comunidade para a atuação na atenção primária. Os menores percentuais de encaminhamentos destes profissionais estão de acordo com os princípios da organização do sistema de saúde em níveis de complexidade, em que $85-95 \%$ das queixas são resolvidas na atenção primária. Estes dados são importantes para os gestores municipais no Brasil perceberem o impacto financeiro e de qualidade dos médicos com formação em MFC atuando na atenção primária. Somente em Florianópolis, no ano de 2016, em que foram coletados estes dados, a diferença no número absoluto de encaminhamentos entre os médicos com residência em MFC e os sem residência foi de 5.420 encaminhamentos, o que equivale a $15 \%$ do total. Considerando os dados levantados pelo recente estudo dos custos da atenção básica em Florianópolis, realizado pela Organização Pan-americana da Saúde (OPAS) ${ }^{32}$, cada consulta nas policlínicas municipais, onde funciona a maior parte da atenção secundária do município, tem um custo de 125 reais, dessa forma, somente o valor das consultas geradas por esses encaminhamentos representaria um impacto financeiro de 682 mil reais no orçamento anual, além de todos os exames solicitados e medicamentos prescritos 
nestas consultas e nas subsequentes na atenção especializada.

Extrapolando-se esses dados para o nível nacional, em que a grande maioria dos médicos que atua na APS não possui nenhuma formação em MFC, podemos imaginar o impacto de um alto percentual de encaminhamentos para o sistema de saúde, sobrecarregando os níveis secundário e terciário com um alto número de pacientes, que muitas vezes poderiam ser acompanhados exclusivamente na atenção primária, fazendo com que pacientes que realmente necessitem de atenção especializada, não cheguem ao especialista, gerando um aumento expressivo dos custos do sistema de saúde.

Além disso, como não houve diferença entre o percentual de encaminhamentos e a proporção de encaminhamentos para as diferentes especialidades entre os médicos com e sem a titulação em MFC, colocando-se em dúvida os critérios considerados para a obtenção do título da especialidade.

No futuro, seria interessante pesquisar outros fatores que podem influenciar o percentual de encaminhamentos, como tempo do médico na mesma equipe, tempo de formado, renda e número de comorbidades da população. Também seria de grande interesse pesquisar a proporção de encaminhamentos adequados entre os médicos com e sem formação em MFC. A pesquisa de indicadores de qualidade relacionada à formação em MFC também seria de fundamental importância, como número de internações por causas sensíveis à atenção primária nas populações assistidas por médicos com formação em MFC versus médicos sem essa formação. Bem como indicadores de custo do cuidado dos pacientes assistidos por médicos com formação em MFC, como número de medicamentos prescritos, número de exames solicitados, comparando com médicos sem essa formação.

\section{Colaboradores}

RC Rebolho foi o responsável pela concepção do trabalho, revisão da literatura, coleta e análise de dados e redação do manuscrito. P Poli Neto contribuiu com a revisão bibliográfica, concepção do trabalho e revisou a versão final a ser publicada. LA Pedebôs contribuiu com a coleta e análise de dados e redação do manuscrito final. LP Garcia contribui com a análise de dados e redação do manuscrito final. AC Vidro contribuiu com a concepção do trabalho, análise de dados e revisou a versão final a ser publicada. 


\section{Referências}

1. Starfield B. Primary Care and Health. JAMA [Internet]. 1991 Oct 23 [cited 2017 Apr 2];266(16):2268. Available from: http://jama.jamanetwork.com/article. aspx?doi=10.1001/jama.1991.03470160100040

2. Starfield B. Primary care Is primary care essential? Lancet [Internet]. 1994 [cited 2017 May 25];344(22):1129-1133. Available from: http://ac.els-cdn.com/S0140673694906343/1-s2.0-S0140673 694906343-main.pdf?_tid=c3fafab6-4192-11e7-8ded-00000aacb35f\&acdnat $=1495748606 \_c b 46 e 76 b 91$ b2326a5c0201e92dd0f0a4

3. Coulter A. Managing demand at the interface between primary and secondary care. $B M J$ [Internet]. 1998 Jun 27 [cited 2017 May 25];316(7149):1974-1976. Available from: http://www.ncbi.nlm.nih.gov/pubmed/9641944

4. Brasil. Ministério da Saúde (MS). Portaria $n^{\circ} 2.436$, de 21 de setembro de 2017. Diário Oficial da União 2017; 22 set.

5. Macinko J, Starfield B, Shi L. Quantifying the Health Benefits of Primary Care Physician Supply in the United States. Int J Heal Serv [Internet]. 2007 Jan 22 [cited 2018 Jan 28];37(1):111-126. Available from: http://journals.sagepub.com/doi/10.2190/3431-G6T7-37M8-P224

6. Brasil. Ministério da Educação (MEC). Secretaria de Educação Superior. Resolução ${ }^{\circ}{ }^{1}$, de 25 de maio de 2015. Diário Oficial da União 2015; 26 maio.

7. DEMOGRAFIA MÉDICA NO BRASIL 2015. [cited 2018 Jan 28]; Available from: https://www.usp. br/agen/wp-content/uploads/DemografiaMedica30nov2015.pdf

8. Andrade MP, Chaves ITS, Zonta R, Silveira JPM. $O$ Serviço Público de Saúde em Florianópolis [apresentação de slides]. Florianópolis: Secretaria Municipal de Saúde de Florianópolis; 2016.

9. Franks P, Williams GC, Zwanziger J, Mooney C, Sorbero M. Why do physicians vary so widely in their referral rates? J Gen Intern Med [Internet]. $2000 \mathrm{Mar}$ [cited 2017 Apr 1];15(3):163-168. Available from: http:// www.ncbi.nlm.nih.gov/pubmed/10718896

10. Barnett ML, Song Z, Landon BE. Trends in physician referrals in the United States, 1999-2009. Arch Intern Med [Internet]. 2012 Jan 23 [cited 2017 Apr 1];172(2):163-70. Available from: http://www.ncbi. nlm.nih.gov/pubmed/22271124

11. Shadd J, Ryan B, Maddocks H, Thind A. Patterns of referral in a Canadian primary care electronic health record database: retrospective cross-sectional analysis. J Innov Heal Informatics [Internet]. 2011 [cited 2017 Apr 1];19(4):217-223. Available from: https://hijournal.bcs.org/index.php/jhi/article/view/816/828

12. Franks P, Zvanziger J, Mooney C, Sorbero M. Variations in Primary Care Physician Referral Rates. HSR Heal Serv Res [Internet]. 1999 [cited 2017 Apr 1];34(1):323-9. Available from: https://www.ncbi. nlm.nih.gov/pmc/articles/PMC1089004/pdf/hsresearch00017-0125.pdf
13. Liddy C, Singh J, Kelly R, Dahrouge S, Taljaard M, Younger J. What is the impact of primary care model type on specialist referral rates? A cross-sectional study. BMC Fam Pract [Internet]. 2014 Feb 3 [cited 2017 Feb 14];15:22. Available from: http://www.ncbi.nlm. nih.gov/pubmed/24490703

14. Ringberg U, Fleten N, Deraas TS, Hasvold T, Førde O. High referral rates to secondary care by general practitioners in Norway are associated with GPs' gender and specialist qualifications in family medicine, a study of 4350 consultations. BMC Health Serv Res [Internet]. 2013 Apr 23 [cited 2017 Feb 14];13:147. Available from: http://www.ncbi.nlm.nih.gov/pubmed/23617296

15. Zielinski A, Håkansson A, Jurgutis A, Ovhed I, Halling A. Differences in referral rates to specialised health care from four primary health care models in Klaipeda, Lithuania. BMC Fam Pract [Internet]. 2008 Nov 26 [cited 2017 Feb 14];9:63. Available from: http:// www.ncbi.nlm.nih.gov/pubmed/19032796

16. O'Donnell CA. Variation in GP referral rates: what can we learn from the literature? Fam Pract [Internet] 2000 Dec 1 [cited 2017 May 25];17(6):462-471. Available from: https://academic.oup.com/fampra/article -lookup/doi/10.1093/fampra/17.6.462

17. Chan BTB, Austin PC. Patient, Physician, and Community Factors Affecting Referrals to Specialists in Ontario, Canada A Population-Based, Multi-Level Modelling Approach. [cited 2018 Jan 27]; Available from: https:// insights.ovid.com/pubmed?pmid=12665714

18. Ryan BL, Shadd J, Maddocks H, Stewart M, Thind A, Terry AL. Methods to Describe Referral Patterns in a Canadian Primary Care Electronic Medical Record Database: Modelling Multilevel Count Data. J Innov Heal Informatics [Internet]. 2017 Nov 17 [cited 2018 Jan 27];24(4):311. Available from: https://hijournal. bcs.org/index.php/jhi/article/view/888

19. Liddy C, Arbab-Tafti S, Moroz I, Keely E. Primary care physician referral patterns in Ontario, Cana$d a$ : a descriptive analysis of self-reported referral data. [cited 2018 Jan 27]; Available from: https:// www.ncbi.nlm.nih.gov/pmc/articles/PMC5567435/ pdf/12875_2017_Article_654.pdf

20. Fleming D. The European Study of referrals from primary to secondary care [Internet]. 1993 [cited 2018 Feb 3]. Available from: https://www.nivel.nl/sites/default/files/bestanden/1001281.pdf

21. Santa Catarina. Secretaria de Saúde. Weblet Importer [Internet]. [cited 2018 Jan 28]. Available from: http:// www.pmf.sc.gov.br/sistemas/saude/unidades_saude/ populacao/uls_2015_index.php

22. Sociedade Brasileira de Medicina da Família e Comunidade (SBMFC). Titulados TEMFC [Internet]. [cited 2018 Jan 28]. Available from: http://www.sbmfc. org.br/default.asp?site_Acao $=$ mostraPagina\&paginaId $=18$

23. Conselho Regional de Medicina de Santa Catarina (CREMESC). Busca Médico. [Internet]. [cited 2018 Jan 28]. Available from: http://www.cremesc.org.br/ buscamedico.jsp 
24. Sistema da Comissão Nacional de Residência Médica (SisCNRM). Consulta de Certificados. [Internet]. [cited 2018 Jan 28]. Available from: http://siscnrm.mec. gov.br/certificados

25. Hosmer D, Lemeshow S. Applied Logistic Regression. $2^{\text {nd }}$ ed. Hoboken: Wiley-Interscience; 2000.

26. $\mathrm{R}$ Core Team. $R$ - A language and environment for statistical computing. Vienna: R Foundation Statistical Computing; 2016.

27. RStudio Team. RStudio: Integrated Development for $R$. Boston: RStudio, Inc; 2016.

28. Knottnerus JA, Joosten J, Daams J. Comparing the quality of referrals of general practitioners with high and average referral rates: an independent panel review. Br J Gen Pract [Internet]. 1990 May [cited 2017 May 25];40(334):178-181. Available from: http:// www.ncbi.nlm.nih.gov/pubmed/2114131

29. Gwynne M, Page C, Reid A, Donahue K, Newton W. What's the Right Referral Rate? Specialty Referral Patterns and Curricula Across I3 Collaborative Primary Care Residencies. [cited 2018 Jan 27]; Available from: http://www.stfm.org/Portals/49/Documents/FMPDF/FamilyMedicineVol49Issue2Gwynne91.pdf

30. Shea D, Stuart B, Vasey J, Nag S. Medicare Physician Referral Patterns. HSR Heal Serv Res [Internet]. 1999 [cited 2018 Jan 27];34(1). Available from: https:// www.ncbi.nlm.nih.gov/pmc/articles/PMC1089005/ pdf/hsresearch00017-0132.pdf

31. Jaturapatporn D, Hathirat S. Does family medicine training affect the referral pattern of primary care doctors in Thailand? Fam Med 2006; 38(6):387-388.

32. Organização Pan-Americana da Saúde (OPAS). Centro de custos da Prefeitura Municipal de Florianópolis [Internet]. 2017 [cited 2018 Jan 28]. Available from: http://189.28.128.100/dab/docs/portaldab/documentos/gestao_custos_dab_abril_2017.pdf

Artigo apresentado em 10/03/2019

Aprovado em 07/06/2019

Versão final apresentada em 09/06/2019

Editores-chefes: Romeu Gomes, Antônio Augusto Moura da Silva 